\title{
Synthesis and application of new rhodamine 6G-derivative fluorescent probe molecules
}

\author{
Chen Wang, Lei Wang, Liansheng Yu, Hengxin Ren, and Yuguang Lv* \\ College of Pharmacy, Jiamusi University, Jiamusi154000, Heilongjiang, PR China
}

\begin{abstract}
In the text, a new fluorescent probe based on rhodamine spirolactam ring was designed and synthesized. Rhodamine-like derivatives have not only good response speed and luminous performance, but also have a special switch type structure, which can specifically recognize responsive metal ions. In the paper, $\mathrm{Rh}-\mathrm{TEF}$, a $6 \mathrm{G}$ derivative of rhodamine, was synthesized by condensation reaction, the synthesized fluorescent probe was successfully used for solutions sample detection.

Keywords: Rhodamine derivatives; Probes; Mercury ion; Sample detection.
\end{abstract}

\section{Introduction}

In recent years, fluorescent molecular-type probes have received a lot of attention because of their good selectivity, no reference term, high sensitivity, relatively simple operation procedure, low preparation costs, and at the same time they are not susceptible to the influence of magnetic and electric fields [1-3]. Fluorescent probe materials with a special switch type structure, rhodamine-type dyes, when the rhodamine ring is in open loop state, its fluorescence emitted by it can be found in the visible light region, and has the advantages of strong emission of fluorescence, rapid response time, etc. And when it is in the closed-loop state, almost no fluorescence is produced, and when the response ion is added, the endospiroamide ring of the fluorescent probe molecule is broken and then fluorescence is produced, based on this mechanism, the application of rhodamine class in fluorescent molecular probes has attracted the attention of many scholars [4-5]. The limit content of elemental mercury in drinking water in China is $1 \mu \mathrm{g} / \mathrm{L}$, and in the field of drinking water purification, the design of rhodamine fluorescent probe can meet the standard of detection limit [6]. At present, there have been many reports about the mercury ion fluorescent probe designed on the principle of the "off-open" mechanism, however, many of the synthesized probes are known to have shortcomings such as low sensitivity, poor selectivity, and limited application range in the detection process [7-11].

In the text, the design, synthesis, optical property exploration and application of rhodamine-derived "off-open" fluorescent probes were carried out as follows: firstly, the designed probe molecules were characterized by mass spectrometry, H-NMR and IR to determine the fluorescent probes in different water bodies was further tested. Based on the data of this experiment and the combination of the structural features of the compound and

* Corresponding author: yuguanglv@163.com 
the literature reviewed to speculate on the response principle of the fluorescent system and to analyze the spectral characteristics of the probe system, it can be concluded that the constructed $\mathrm{Hg}^{2+}$ fluorescent probe based on the structure of the rhodamine derivative spirocycles can be used for environmental water quality monitoring in this experiment.

\section{Experiments}

\subsection{Synthesis of Rhodamine 6G derivatives like Rh-TEF}

Synthesis of intermediate rhodamine 6G hydrazide:In methanol with rhodamine 6G, add hydrazine hydrate dropwise, heated back to reflux for $4 \mathrm{~h}$ until the liquid color of the reaction solution became almost clear, cooled to room temperature and then rotated to evaporate the methanol, washed with distilled water to obtain the crude product, and dried under vacuum to obtain a pink solid.

Synthesis of Rh-TEF: Take the synthesized rhodamine 6G hydrazide into methanol and add an excess of triethyl orthoformate, reflux for $6 \mathrm{~h}$. When the color of the liquid changes from the initial red to purple, stop the reflux heating, cool to room temperature, reduce the pressure and evaporate to half of the reaction solution, and rinse the filter cake repeatedly with distilled water after filtration. The solid obtained is Rh-TEF.

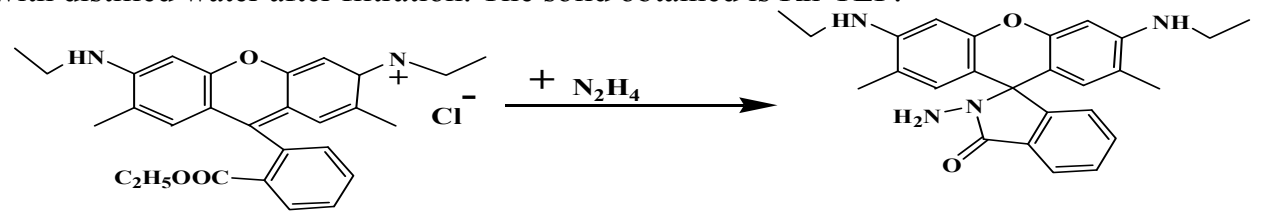

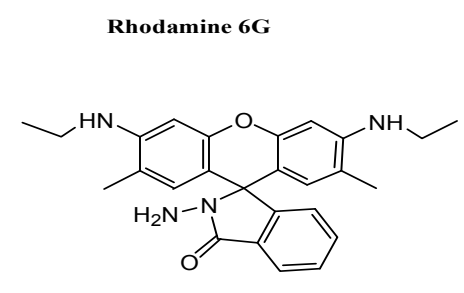

Rhodamine 6G hydrazide

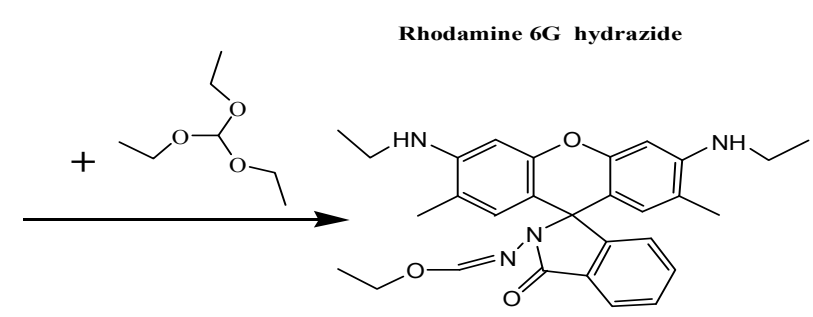

Rh-TEF

Fig. 1. Rh-TEF synthesis.

\section{Results and discussion}

\subsection{Synthesis results of Rh-TEF a rhodamine $6 \mathrm{G}$ derivative}

Analysis of the results of nuclear magnetic measurements: in the NMR hydrogen spectrum of rhodamine 6G hydrazide (Figure 2), there is a single peak at a chemical shift of 3.590, where there are two hydrogens, indicating the formation of a $\mathrm{C}-\mathrm{N}$ bond i.e., the successful synthesis of rhodamine $6 \mathrm{G}$ hydrazide. In the nuclear magnetic hydrogen spectrum of rhodamine-like Rh-TEF (Figure 3), there are two triple peaks at chemical shifts 1.603, 1.6381 , where there are three hydrogens, i.e., the $-\mathrm{OCH}_{2} \mathrm{CH}_{3}$ structure is already present in the compound; and at the chemical shift of 7.992, there is a single peak, where there is one hydrogen, i.e, the intermediate structure is connected with $\mathrm{N}=\mathrm{CH}$, i.e, $\mathrm{Rh}-\mathrm{TEF}$ is successfully synthesized. 


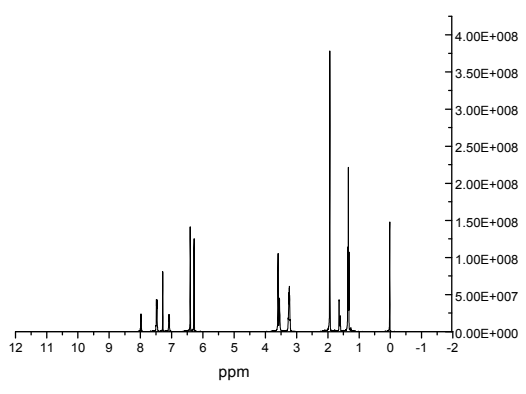

Fig. 2. NMR spectra of Rh-TEF intermediates.

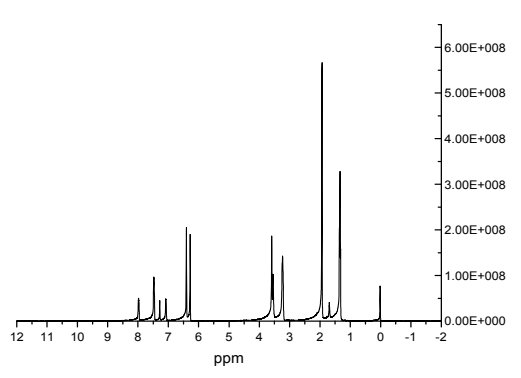

Fig. 3. NMR hydrogen spectrum of Rh-TEF.

Analysis of the results of IR spectroscopy: in the infrared spectrogram of rhodamine 6G hydrazide (Figure 4), there is a strong absorption peak at $3427 \mathrm{~cm}^{-1}$, which is the telescopic vibration of the $\mathrm{N}-\mathrm{H}$ bond; the absorption peak at $3012 \mathrm{~cm}^{-1}$ is the absorption peak of the Ar- $\mathrm{H}$ of the aromatic ring, in addition, at $1516 \mathrm{~cm}^{-1}$ is the telescopic vibration of the $\mathrm{C}=\mathrm{C}$ of the aromatic ring structure, which can determine the presence of the structure; at $1688 \mathrm{~cm}^{-1}$ is the telescopic vibration of the $\mathrm{C}=\mathrm{O}$; and a strong absorption peak at $1269 \mathrm{~cm}^{-1}$ is the telescopic vibration of the $\mathrm{C}-\mathrm{O}$ structure, which can determine the presence of the structure.In the infrared spectrum of rhodamine-derived Rh-TEF (Figure 5), it is possible to identify the absorption peak of the characteristic functional group of the product Rh-TEF containing the intermediate, and at the same time the intermediate rhodamine $6 \mathrm{G}$ hydrazide in the basis, there is an absorption peak at $1622 \mathrm{~cm}^{-1}$, which is the telescopic vibration peak of $\mathrm{C}=\mathrm{N}$, and $\mathrm{Rh}-\mathrm{TEF}$ has a strong absorption peak at this position, the results show that by the The $-\mathrm{NH}_{2}$ structure of rhodamine $\mathrm{B}$ hydrazide forms a new $(\mathrm{C}=\mathrm{N})$ bond with the $\mathrm{C}=\mathrm{O}$ structure; an absorption peak at $2871 \mathrm{~cm}^{-1}$ is the structure $-\mathrm{OCH}_{2} \mathrm{CH}_{3}$, and in summary, it can be judged that Rh-TEF is successfully synthesized.

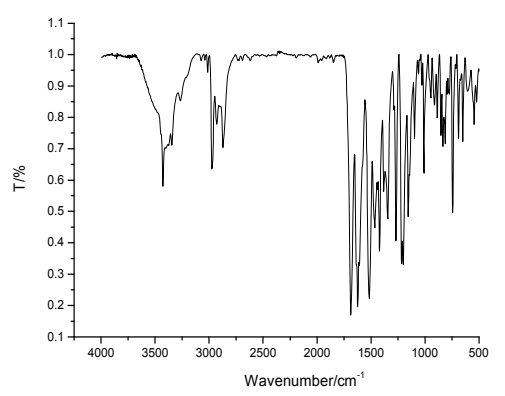

Fig. 4. Infrared spectra of Rh-TEF intermediates.

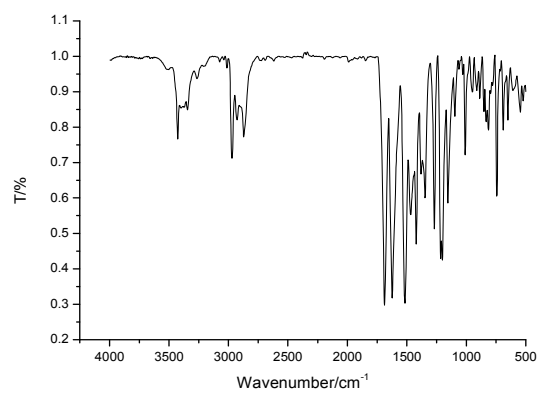

Fig. 5. Infrared spectrum of Rh-TEF.

Mass spectrometry results: the mass spectra of the rhodamine derivative class Rh-TEF (Figure 6) show that the measured value of $[\mathrm{M}+\mathrm{H}]+$ is 485 , which is consistent with the theoretical calculated value of 485 . 


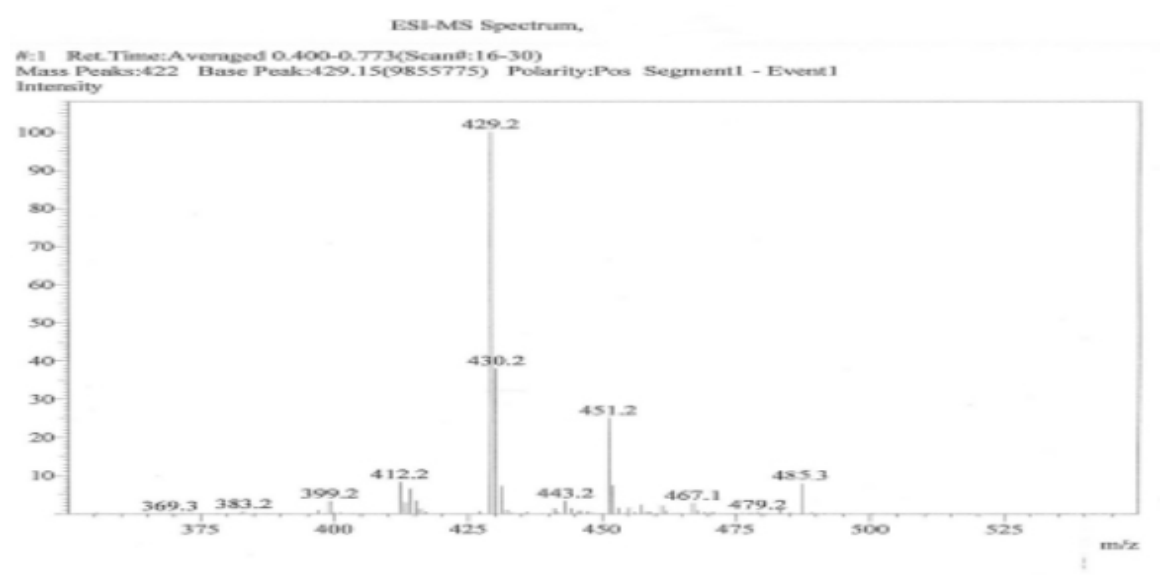

Fig. 6. Mass spectrogram of Rh-TEF.

\subsection{Rhodamine 6G derivatives Rh-TEF probe sample results}

Table 1. Water sample ion detection.

\begin{tabular}{ccccc}
\hline Sample & $\begin{array}{c}\text { Detection } \\
\text { Volume(mol/L) }\end{array}$ & $\begin{array}{c}\text { Add Amount } \\
(\mathrm{mol} / \mathrm{L})\end{array}$ & $\begin{array}{c}\text { Recovery (\%) } \\
\text { Rate }\end{array}$ & $\begin{array}{c}\text { RSD } \\
(\%)\end{array}$ \\
\hline \multirow{3}{*}{ Tap water } & -- & $10 \times 10^{-6}$ & $99.5 \%$ & 1.89 \\
& & $20 \times 10^{-6}$ & $100.8 \%$ & 2.05 \\
& & $40 \times 10^{-6}$ & $101.1 \%$ & 1.96 \\
Songhua & & $10 \times 10^{-6}$ & $98.7 \%$ & 2.13 \\
River & -- & $20 \times 10^{-6}$ & $100.9 \%$ & 1.98 \\
water & & & & \\
& & $40 \times 10^{-6}$ & $101.8 \%$ & 1.85 \\
\hline
\end{tabular}

Table 1 shows the experiment for the determination of mercury ions in water samples. The river water and tap water are simply filtered and heated through the filter membrane, and then the prepared fluorescent probe is used to measure the mercury ion content. There is no fluorescence, indicating that there is no $\mathrm{Hg}^{2+}$; there are three different $\mathrm{Hg}^{2+}$ spikes in high, medium and low. It maintains a high similarity with the measured data, indicating that the probe RH-TEF can effectively analyze and determine the presence or absence of $\mathrm{Hg}^{2+}$ in tap water and river water.

\section{Conclusion}

In the text, the structure of Rhodamine $6 \mathrm{G}$ was used as the parent for structural modification, and a new fluorescent probe of rhodamine was designed and synthesized. The results were characterized by nuclear magnetic resonance, infrared spectroscopy, and mass spectrometry, and the product was determined to be the ideal product, the new rhodaminebased Rh-TEF Probe molecule. The detection results of river water and tap water show that the new rhodamine-based Rh-TEF probe molecules have good analysis and detection functions for $\mathrm{Hg}^{2+}$ in water samples, and can be applied to the detection of $\mathrm{Hg}^{2+}$ in water samples in the future. 


\section{Acknowledgements}

This work was financially supported by the National Science Foundation of China (No.21346006), Department of scientific research project in Heilongjiang province (No. B2017015), Excellent discipline team project of Jiamusi University ((No. JDXKTD2019007).

\section{References}

1. Qiao L., Yang H., Hai-Nan H., 2018. A new coumarin-carbonothioate-based turn-on fluorescent chemodosimeter for selective detection of $\mathrm{Hg}^{2+}$. Inorganica Chimica Acta, 471(24), pp.705-708.

2. Haixing L., Qing L., Yanfu S.,2017. Research progress based on rhodamine-based fluorescent probes. Modern Chemical Engineering,37(04), pp.197-204.

3. Serkan Erdemir., 2017. Ozcan Kocyigit.A novel dye based on phenolphthaleinfluorescein as a fluorescent probe for the dual-channel detection of $\mathrm{Hg}^{2+}$ and $\mathrm{Zn}^{2+}$. Dyes and Pigments, 145, pp.72-79.

4. Dmitry S., 2020. Koktysh. Ratiometric pH sensor using luminescent $\mathrm{CuInS}_{2} / \mathrm{ZnS}$ quantum dots and fluorescein. Materials Research Bulletin, 123, pp.110686.

5. Shohei Yamaguchi., Kazuhiro Shimada., Kazunori Matsui., 2020. Spectroscopic study of fluorescein immobilized on anodic porous alumina in aqueous solutions of different pH. Dyes and Pigments, 173, pp.107944.

6. Zhenzhen Z., Yongmei W., Shizhen H., 2018. Ratiometric fluorescence sensing of mercuric ion based on dye-doped lanthanide coordination polymer particles. Analytica Chimica Acta, 1014(19), pp.85-90.

7. Cuicui J., Mian W., Yanxiu W., 2017. Synthesis and evaluation of two novel rhodamine-based fluorescence probes for specific recognition of $\mathrm{Fe}^{2+}$ ion. Tetrahedron Letters, 58(26), pp.2560-2565.

8. Rina Komatsu., Tamayo Yamaguchi., Naohiro Kobayashi., 2018. Synthesis of alkynetagged and biotin-tagged Sortin1 as novel photoaffinity probes. Bioorganic\&Medicinal Chemistry Letters, 28(9), pp.1562-1565.

9. Ting D., Ling L., Bo Z., 2017. Progress in the preparation and application of rhodamine-like mercury ion fluorescent probes. Modern Salt Chem, 44(05), pp.18-21.

10. Jiaqin W., Ling L., Ting D., 2017. Preparation and properties of a mercury ion fluorescent probe with rhodamine structure. Energy Chem, 38(06), pp.48-53.

11. Yingchun G., Dayong L., Xuening F., 2019. Labeling of Microthrix parvicella in situ: A novel FRET probe based on bisoctyl rhodamine B. Spectrochimica Acta Part A: Molecular and Biomolecular Spectroscopy, 213(15), pp.263-271. 